NBER WORKING PAPER SERIES

\title{
PERMANENT AND TRANSITORY COMPONENTS \\ IN MACROECONOMIC FLUCTUATIONS
}

\author{
John Y. Campbe 11
}

N. Gregory Mankiw

Working Paper No. 2169

NATIONAL BUREAU OF ECONOMIC RESEARCH

1050 Massachusetts Avenue

Cambridge, MA 02138

February 1987

The research reported here is part of the NBER's research program in Economic Fluctuations. Any opinions expressed are those of the authors and not those of the National Bureau of Economic Research. 
NBER Working Paper \#2169

February 1987

Permanent and Transitory Components in Macroeconomic Fluctuations

\section{ABSTRACT}

Fluctuations in real GNP have traditionally been viewed as transitory deviations from a deterministic time trend. The purpose of this paper is to review some of the recent developments that have led to a new view of output fluctuations and then to provide some additional evidence. Using post-war quarterly data, it is hard to reject the view that real GNP is as persistent as a random walk with drift.

We also consider the hypothesis that the recent finding of persistence are due to the failure to distinguish the business cycle from other fluctuations in real GNP. We use the measured unemployment rate to decompose output fluctuations. We find no evidence for the view that business cycle fluctuations are more quickly trend-reverting.

John Y. Campbe 11

NBER

1050 Massachusetts Avenue

Cambridge, MA 02138
N. Gregory Mankiw NBER 1050 Massachusetts Avenue Cambridge, MA 02138 


\section{Permanent and Transitory Components in Macroeconomic Fluctuations}

John Y. Campbe 11 and N. Gregory Mankiw*

A "stylized fact" is sometimes defined as an empirical claim that is widely believed but the evidence for which is only mixed. Seen in this light, it is perhaps not surprising that one of the principal stylized facts of macroeconomics has undergone a substantial reexamination over the past five years. The purpose of this paper is to review some of the recent developments that have led to the new view of output fluctuations and then to provide some additional evidence.

According to the traditional view, fluctuations in real GNP primarily reflect temporary deviations of production from trend. Only a few years ago, economists as diverse as Olivier Blanchard (1981) and Finn Kydland and Edward Prescott (1980) subscribed to the then uncontroversial suggestion that the logarithm of quarterly real GNP is well represented as a stationary second-order autoregressive process around a deterministic time trend. Blanchard estimated the following process for detrended log real GNP:

$$
y_{t}=1.34 y_{t-1}-0.42 y_{t-2}+\epsilon_{t} \text {. }
$$

This AR(2) process implies that the effect of a shock to output increases for a few quarters, but the effect dies out soon thereafter. Only 8 percent of a shock to output remains after 20 quarters. 1 These authors, along with many others, viewed this dynamic response of output to an innovation as an important phenomenon to be explained by macroeconomic theory. The reexamination of this traditional view was motivated in part by 
developments in the econometrics of non-stationary time series (e.g., David Dickey and wayne fuller, 1981). In one of the first applications of the new econometric techniques to standard macroeconomic data, Charles Nelson and Charles plosser (1982) argued that most of these time series, including real GNP, are not stationary around a deterministic trend. Instead, these series should be considered stationary only after differencing. Nelson and Plosser suggested that the data for output are well approximated by simple stochastic processes that imply no trend reversion at all.

Much disagreement remains over exactly how persistent are shocks to output. Nonetheless, among investigators using post-war quarterly data, there is almost unanimity that there is a substantial permanent effect. In a previous paper (1986), we estimated unconstrained ARMA models for GNP growth. We found that a negative one percent innovation lowers the level of GNP by over one percent over any foreseeable horizon. Moreover, when the non-parametric procedure suggested by John Cochrane (1986) is applied to this data, the same finding is obtained. Authors using unobserved components models (Mark Watson, 1986; Peter Clark, 1987) have typically found less persistence. Even so, the long-run impact of a shock to GNP is usually estimated to be about 0.6 . Hence, almost all recent studies have rejected the traditional view that output shocks have little or no permanent effect.

\section{Two Concepts of Persistence}

What is persistence? For some purposes, a shock to an economy may be considered persistent if it lasts for more than one period. Here, however, we take persistence as meaning "continuing for a long time into the future." More formally, suppose that the change in $\log$ of GNP is a stationary process with moving average representation 


$$
\Delta Y_{t}=A(L) \epsilon_{t}
$$

where $A(L)$ is an infinite polynomial in the lag operator, and $\epsilon_{t}$ is white noise. The impact of a shock in period $t$ on the growth rate in period $t+k$ is $A_{k}$. The impact of the shock on the level of GNP in period $t+k$ is therefore $A_{0}+A_{1}+\ldots+A_{k}$. The ultimate impact of the shock on the level of GNP equals the infinite sum of these moving average coefficients, which is $A(1)$. The value of $A(1)$ is the measure of persistence we used in our previous paper. For a random walk, $A(1)$ equals one; for any series stationary around a deterministic trend, $A(1)$ equals zero.

Cochrane has recently proposed another measure of persistence. His measure can be written either as a ratio of variances or as a function of autocorrelations:

$$
\frac{1}{k+1} \frac{\operatorname{Var}\left(Y_{t+k+1}-Y_{t}\right)}{\operatorname{Var}\left(Y_{t+1}-Y_{t}\right)} \equiv 1+2 \sum_{j=1}^{k}\left(1-\frac{j}{k+1}\right) \rho_{j}
$$

where $\rho_{j}$ is the $j$-th autocorrelation of $\Delta Y_{t}$. If $Y_{t}$ follows a random walk, then the variance of the $(k+1)$-lagged difference is $(k+1)$ times the variance of the once-lagged difference. Hence, for a random walk, the above ratio is one. For any stationary series, the variance of the $(k+1)$-lagged difference approaches twice the variance of the series. Hence, for any stationary series, the above ratio approaches zero for large $k$. Cochrane therefore proposes using the limit of the variance ratio as a measure of persistence. We call this limiting variance ratio $v$.

For two simple cases--a stationary process and a random walk--the two measures of persistence produce the same number. More generally, however, the two measures are not the same. Define $R^{2} \equiv 1-\operatorname{Var}(\epsilon) / \operatorname{Var}(\Delta Y)$, the fraction 
of the variance that is predictable from knowledge of the past history of the process. Then $A(1)$ can be expressed as

$$
A(1)=\sqrt{\frac{V}{1-R^{2}}} .
$$

Equation (4) shows that the square root of Cochrane's measure of persistence is a lower bound on $A(1)$. The more highly predictable is the differenced process, the greater is the disparity between the two measures.

\section{Approaches to Estimating Persistence}

At least three general approaches have been proposed for estimating persistence. Here we provide a brief overview.

A. The ARMA Approach

In our previous paper (1986), we modeled the change in log GNP as a stationary ARMA process. That is,

$$
\phi(L) \Delta Y_{t}=\theta(L) \epsilon_{t^{\prime}}
$$

where $\phi(L)$ and $\theta(L)$ are finite polynomials in the lag operator. The moving average representation $A(L)$ equals $\phi(L)^{-1} \theta(L)$, from which we computed the persistence measure $A(1)$.

If $Y_{t}$ is in fact stationary around a deterministic trend, then the ARMA model in equation (5) is overdifferenced. This overdifferencing induces a unit root in the moving average component $\theta(L)$. For the ARMA approach to be able to detect stationarity, it is therefore necessary to allow for the possibility of a unit MA root by including at least one moving average parameter. Long autoregressions do not provide an adequate approximation in 


$$
-5-
$$

this case. Moreover, it is important to avoid estimation of the ARMA model via quasi-maximum likelihood techniques, since these may not provide good approximations when there is a unit MA root. In our previous paper, we estimated all models up to $\operatorname{ARMA}(3,3)$ using exact maximum likelihood.

We concluded that an ARMA $(2,2)$ model well approximates post-war, quarterly, real GNP growth. The ARMA $(2,2)$ model and most of the other models produce similar impulse response functions, $A(L)$. After a unit shock, the maximum impact on the level of real GNP is about 1.6 after a few quarters, followed by a slight (and perhaps insignificant) decline. The long-run impact A(1) was estimated to be about 1.5 .

\section{B. The Nonparametric Approach}

One can estimate the persistence measure $V$ very simply by replacing the population autocorrelations in equation (3) with the sample autocorrelations. 2 The estimator is

$$
\hat{v}^{k} \equiv 1+2 \sum_{j=1}^{k}\left(1-\frac{j}{k+1}\right) \hat{\rho}_{j} .
$$

As long as $k$ increases with the sample size, this estimator consistently estimates $V$.

It is also possible to compute nonparametrically an approximate estimate of $A(1)$, called $\hat{A}^{k}(1)$, as

$$
\hat{A}^{k}(1) \equiv \sqrt{\frac{\hat{V}^{k}}{1-\hat{\rho}_{1}^{2}}} .
$$

The estimate of $A(1)$ is computed by replacing the $R^{2}$ in equation (4) with the square of the first autocorrelation. Since $\rho_{1}^{2}$ is an underestimate of $R^{2}$, except for an $A R(1)$ process, this estimate tends to understate $A(1)$. 
In any given sample, it is of course necessary to choose $k$, the number of autocorrelations to include. Including too few autocorrelations may obscure trend reversion manifested in higher autocorrelations. Including too many autocorrelations may tend to find excessive trend reversion, since as $k$ approaches the sample size $T$, the estimator approaches zero. Since the sample mean has been removed from the data, $\hat{V}^{k}$ is identically zero at $k=T-1$. Hence, while large $k$ appears preferable, $k$ must be small relative to the sample size.

To examine more precisely the choice of window size $(k)$, we have performed a small Monte Carlo experiment. The true process is, alternatively, a random walk with drift or a stationary $A R(2)$ process around a deterministic time trend. The parameters for the AR(2) process are those estimated by Blanchard for detrended real GNP (equation 1). There are 500 replications, and each has 130 observations, which is the number of post-war quarterly observations we use below. Table 1 reports the mean of the two persistence estimates for various $k$, as well as the standard deviation of the estimates.

The results in Table 1 show clearly that the window size $k$ must be small relative to the sample size. For $k=50$, the mean estimate of $V$ for the random walk is 0.59 , in contrast to a population parameter of unity. There is thus severe downward bias for large $k .^{3}$

The results in Table 1 also show how difficult it is to distinguish between the two representations on the basis of these nonparametric persistence estimates. For $k=40$, the mean estimate of $V$ is 0.67 for $a$ random walk and 0.43 for the stationary $A R(2)$. Moreover, the associated standard deviations are substantial. One cannot make inferences from these persistence estimates with great confidence. 
In Table 2 we report $\hat{V}^{k}$ and $\hat{A}^{k}(1)$ for the log of quarterly real GNP from 1952:2 to $1984: 3$ for various values of $k$. The results are consistent with those obtained with the ARMA approach. A comparison of the estimates in Table 2 with the Monte Carlo results in Table 1 shows that real GNP appears more persistent than the stationary $A R(2)$ process in equation (1). For $k=40$, the estimate of $V$ is 0.85 , which is about two standard deviations above the 0.43 mean of the $A R(2)$ process. Similarly, the estimate of $A(1)$ of 0.98 is about one standard deviation above the 0.69 mean of the $A R(2)$. Indeed, the estimates for real GNP slightly exceed the mean persistence estimates for a random walk.

C. The Unobserved Components Approach

An alternative way to model output is as the sum of two or more components. These components are not directly observed, but their relative importance, and the implications for persistence, are inferred from the time series behavior for output. Authors such as Beveridge and Nelson (1981), Watson, and Clark have used this approach.

Obviously some restrictions must be imposed on the components if they are to be identifiable from a single time series for output. Beveridge and Nelson proposed a decomposition into two components, a random walk and a stationary component, whose innovations are perfectly correlated. For any output process, these components are just identified.

The two measures of persistence can be interpreted in terms of the Beveridge-Nelson decomposition. Cochrane points out that the measure $V$ is the variance of the change in the random walk component divided by the variance of the total change in output. It follows from equation (4) that the 
measure $A(1)$ is the standard deviation of the change in the random walk component divided by the standard deviation of the univariate innovation to output. It is tempting to conclude that $V$ and $A(1)$ convey information about the relative importance of "trend" and "cycle" components in output growth. But of course, one usually thinks of trend and cycle as having a low or zero correlation, while the Beveridge-Nelson components are perfectly correlated.

An alternative type of unobserved components model, estimated by watson and Clark, expresses output as the sum of two independent processes. The independence of the two components implies that the measure $v$ can be written as a weighted average of the equivalent measures for the two components. Formally, if $v_{1}$ and $v_{2}$ are the persistence of components 1 and 2 , and the changes in these components have variances $\sigma_{1}^{2}$ and $\sigma_{2}^{2}$, then

$$
v=\lambda v_{1}+(1-\lambda) v_{2}
$$

where $\lambda=\sigma_{1}^{2} /\left(\sigma_{1}^{2}+\sigma_{2}^{2}\right)=\sigma_{1}^{2} / \operatorname{Var}(\Delta Y)$.

Watson and clark assume that the first component is a random walk and the second component is a stationary $A R(2)$, so that $V_{1}=1$ and $V_{2}=0$. Since the sum of a random walk and an $\operatorname{AR}(2)$ process can be represented as an $\operatorname{ARMA}(2,2)$ process in the growth rates, the watson-clark model can be viewed as imposing restrictions on this ARMA model. As in the Beveridge-Nelson decomposition, $V$ equals the share of the random walk in output variability. Note, however, that $V$ and $A(1)$ must now be no greater than unity to be consistent with the model. Watson and clark thus rule out highly persistent processes a priori.

Watson and clark estimate the parameters of the unobserved components model via maximum $l i k e l i h o o d$ and then infer $V$ and $A(1)$. Watson, for example, 
finds that $V=0.36$ and $A(1)=0.57$, suggesting that both the permanent and transitory components of GNP are important. Clark's results are comparable.

\section{Using the Unemployment Rate to Measure the Business Cycle}

The unobserved components models, in contrast to the unrestricted ARMA models or the non-parametric approach, suggest that there is a substantial temporary component to real GNP. The results with these models, therefore, are easier to reconcile with standard theories of the business cycle. One is tempted to interpret the permanent component as the natural rate of output, and the temporary component as the deviation of output from the natural rate. Indeed, the appeal of the restrictions imposed by Watson and Clark comes largely from the long tradition of separating issues of long-run growth from issues of short-run fluctuations.

The purpose of this section is to examine whether one can decompose output fluctuations into a transitory component associated with the business cycle and a persistent component unrelated to the business cycle. The decompositions of Watson and Clark are completely univariate. In contrast, we use the unemployment rate as a measure of the business cycle. 4

We begin by examining the persistence of fluctuations in the rate of unemployment. Table 3 presents estimates of $V$ and $A(1)$ computed as in equations (6) and (7) using quarterly data from 1952:2 to 1984:3. The results for $V$ indicate that shocks to unemployment are no more persistent than the $A R(2)$ process simulated in Table 1 . Yet since $\rho_{1}$ is much larger for unemployment, the estimates of $A(1)$ are larger.

We next use the unemployment rate to separate the cyclical and trend components of real GNP. As in the unobserved components models discussed 
above, we make the identifying assumption that these two components are uncorrelated. In contrast to these models, we do not assume the two components are unobserved. Instead, we assume that the cyclical component is that part of GNP correlated with unemployment at leads and lags, while the trend component is that part of GNP uncorrelated with unemployment. Hence, one might refer to our decomposition as an "observed components model."

We accomplish this decomposition by regressing the change in log real GNP on eight leads, eight lags, and the contemporaneous detrended unemployment rate. This regression yields an $R^{2}$ of about 0.6 . We take the fitted value of this regression as a measure of the change in the cyclical component of real GNP. The residual is a measure of the change in the trend component.

Table 4 presents estimates of the persistence of the cyclical and trend components of real GNP. The persistence of the two components of GNP appears roughly equal. Indeed, to the extent that the two components differ, the cyclical component of GNP appears somewhat more persistent.

These results call into question the identifying assumptions underlying unobserved components models. These models are based on the premise that fluctuations in output associated with the business cycle are less persistent than other fluctuations in output. Yet when the unemployment rate is used as a measure of the business cycle, it is hard to find a significant difference in the persistence of these two components.

If one imposes some restrictions on the regression decomposing GNP growth, it is possible to find some evidence for the view that the cyclical component is less persistent than the trend component. In particular, including fewer leads and lags of the unemployment rate, or including the 
unemployment rate only in differenced form, tends to make the fitted value of the regression somewhat less persistent. In these cases, the estimated $V$ for large $k$ is smaller for the cyclical component, while the estimated $A(1)$ remains larger for this component. If there were reason to believe these constraints a priori, then the sort of decomposition suggested here might be interpreted as providing weak evidence for the use of unobserved components mode is.

\section{Conclusions}

Not very long ago, it was widely believed that shocks to output almost fully dissipate in four or five years. The research summarized here has challenged that stylized fact. Fluctuations in output appear far more persistent. Indeed, it hard to reject the view that post-war real GNP is as persistent as a random walk with drift.

of course, in any finite set of data, it is impossible to reject the view that output eventually returns to a deterministic time trend. The sort of evidence presented here can only demonstrate that such trend reversion does not occur as quickly as was once believed.

Some economists have dismissed the recent findings of persistence in real GNP because these findings do not distinguish the business cycle from other fluctuations. We have attempted to make such a distinction by using the unemployment rate as a measure of the business cycle. In contrast to what many economists have assumed, fluctuations associated with the business cycle are not obviously more trend-reverting than other fluctuations in output. 
Table 1: Persistence Estimates and the Window Size

\section{A. True Process is a Random Walk}

Window Size $(k)$

10

20

30

40

50

60

75

100

\begin{tabular}{llll}
\multicolumn{3}{c}{ Persistence Measure } \\
\hline \multicolumn{2}{c}{$V$} & 0.94 & $(0.15)$ \\
0.90 & $(0.29)$ & 0.89 & $(0.21)$ \\
0.83 & $(0.39)$ & 0.84 & $(0.25)$ \\
0.76 & $(0.46)$ & 0.78 & $(0.27)$ \\
0.67 & $(0.48)$ & 0.72 & $(0.29)$ \\
0.59 & $(0.49)$ & 0.65 & $(0.30)$ \\
0.51 & $(0.48)$ & 0.56 & $(0.30)$ \\
0.40 & $(0.43)$ & 0.38 & $(0.28)$ \\
0.21 & $(0.30)$ & &
\end{tabular}

\begin{tabular}{|c|c|c|c|c|}
\hline \multirow{3}{*}{$\frac{\text { Window Size }(k)}{10}$} & \multicolumn{4}{|c|}{ Persistence Measure } \\
\hline & \multicolumn{2}{|c|}{$\mathrm{V}$} & & $A(1)$ \\
\hline & 1.32 & $(0.38)$ & 1.24 & $(0.20)$ \\
\hline 20 & 0.83 & $(0.32)$ & 0.97 & $(0.20)$ \\
\hline 30 & 0.57 & $(0.27)$ & 0.80 & $(0.19)$ \\
\hline 40 & 0.43 & $(0.23)$ & 0.69 & $(0.19)$ \\
\hline 50 & 0.34 & $(0.21)$ & 0.60 & $(0.19)$ \\
\hline 60 & 0.27 & $(0.19)$ & 0.53 & $(0.19)$ \\
\hline 75 & 0.21 & $(0.17)$ & 0.45 & $(0.20)$ \\
\hline 100 & 0.11 & $(0.14)$ & 0.31 & $(0.21)$ \\
\hline
\end{tabular}

Note: This table presents the results of a Monte Carlo experiment. It displays the mean of the persistence estimate and, in parentheses, the standard deviation of the estimates. These results are based on a sample of 130 and 500 replications. 


\section{Table 2}

\section{Persistence of Post-War Quarterly Real GNP}

Window size $(k)$

10

20

30

40

50

60

\section{Estimate of $\mathrm{V}$}

$1.43(0.48)$

$1.09(0.50)$

$1.02(0.57)$

$0.85 \quad(0.55)$

$0.64(0.46)$

$0.57 \quad(0.45)$

\section{Estimate of $A(1)$}

1.27

1.11

1.07

0.98

0.85

0.80

Note: Asymptotic standard errors are in parentheses. See Campbell and Mankiw (1986) . 


\section{Table 3}

Persistence of Post-War Quarterly Unemployment Rate

\begin{tabular}{|c|c|c|c|}
\hline Window Size (k) & Estim & te of $V$ & Estimate of $A(1)$ \\
\hline 10 & 1.42 & $(0.47)$ & 1.53 \\
\hline 20 & 0.81 & $(0.38)$ & 1.15 \\
\hline 30 & 0.67 & $(0.38)$ & 1.05 \\
\hline 40 & 0.49 & $(0.32)$ & 0.90 \\
\hline 50 & 0.31 & $(0.23)$ & 0.72 \\
\hline 60 & 0.23 & $(0.18)$ & 0.62 \\
\hline
\end{tabular}

Note: Asymptotic standard errors are in parentheses. See Campbell and Mankiw (1986). 


\section{Notes}

*Princeton University, Princeton N.J. 08544 ; Harvard University,

Cambridge, MA 02138. We are grateful to James Poterba for helpful comments.

1. This dynamic pattern also revealed itself in explanations of the

business cycle. Barro and Rush (1980), for example, estimated that

unanticipated money has its greatest effect with a lag of three quarters, but has no effect after ten quarters.

2. We compute the $j$-th sample autocovariance as the sum of the $T-j$ cross products divided by $T-j$. This computation does not guarantee that the estimate of the variance ratio is positive, however. Dividing the $T-j$ cross-products by $T$ would guarantee a positive estimate. In practice, as long as $k$ is small relative to the sample size, the difference is not important. 3. It appears that, for the random walk, this bias can be approximately corrected by multiplying by $T /(T-k)$.

4. George Evans (1986) also studies persistence in a bivariate model of output and unemployment. His use of restricted and unrestricted vector autoregressions is very different from the approach taken here. 


\section{Table 4}

Persistence of the Cyclical and Trend Components of Real GNP

A. The Cyclical Component

\begin{tabular}{cccc} 
Window Size $(k)$ & \multicolumn{2}{c}{ Estimate of $V$} & Estimate of $A(1)$ \\
\cline { 2 - 3 } 10 & 1.92 & $(0.64)$ & 1.87 \\
20 & 1.25 & $(0.58)$ & 1.51 \\
30 & 1.09 & $(0.61)$ & 1.41 \\
40 & 0.82 & $(0.53)$ & 1.23 \\
50 & 0.55 & $(0.40)$ & 1.00 \\
60 & 0.40 & $(0.32)$ & 0.85
\end{tabular}

B. The Trend Component

Window size $(k)$

10

20

30

40

50

60

\section{Estimate of $\mathrm{V}$}

$0.39 \quad(0.13)$

$0.38 \quad(0.17)$

$0.35 \quad(0.19)$

$0.31 \quad(0.20)$

$0.29 \quad(0.21)$

$0.31 \quad(0.24)$
Estimate of $A(1)$

$$
0.64
$$

0.63

0.61

0.58

0.56

0.57

Note: Asymptotic standard errors are in parentheses. See Campbell and Mankiw (1986). 


\section{References}

Barro, Robert J., and Mark Rush, "Unanticipated Money and Economic Activity," in S. Fischer, ed., Rational Expectations and Economic Policy, Chicago: University of Chicago Press, 1980.

Beveridge, S. and Charles R. Nelson, "A New Approach to Decomposition of Economic Time Series into Permanent and Transitory Components with Particular Attention to Measurement of the 'Business Cycle,'" Journal of Monetary Economics, 1981, $7,151-74$.

Blanchard, Olivier J., "What is Left of the Multiplier-Accelerator?" American Economic Review, May 1981, 150-4.

Campbe11, John Y., and N. Gregory Mankiw, "Are Output Fluctuations Transitory?" NBER Working Paper No. 1916, 1986.

Clark, Peter K., "The Cyclical Component of U.S. Economic Activity," Quarterly Journal of Economics, forthcoming 1987.

Cochrane, John H., "How Big is the Random Walk in GNP?" University of Chicago, 1986.

Dickey, David A., and Wayne A. Fuller, "Likelihood Ratio Statistics for Autoregressive Time Series with a Unit Root," Econometrica, 1981, 42, $1057-72$.

Evans, George W., "Output and Unemployment Dynamics in the United States: 1950-85," Stanford University, 1986.

Kydland, Finn E., and Edward C. Prescott, "A Competitive Theory of Fluctuations and the Feasibility and Desirability of Stabilization Policy," in S. Fischer, ed., Rational Expectations and Economic Policy, Chicago: University of Chicago Press, 1980. 
$-18-$

Nelson, Charles R., and Charles I. Plosser, "Trends and Random Walks in Macroeconomic Time Series," Journal of Monetary Economics, 1982, 10, 139-62.

Watson, Mark W., "Univariate Detrending Methods with Stochastic Trends," Journal of Monetary Economics, 1986, 18, 1-27. 